Noname manuscript No.

(will be inserted by the editor)

\title{
Data Analysis and Phase Ambiguity Removal in the ACES Microwave Link
}

\author{
Loïc Duchayne · Peter Wolf · Luigi Cacciapuoti · \\ Marc-Peter Hess · Marco Siccardi · Wolfgang \\ Schafer
}

Received: date / Accepted: date

\begin{abstract}
The ACES (Atomic Clock Ensemble in Space) mission is an ESA - CNES project with the aim of setting up onboard the International Space Station (ISS) several highly stable atomic clocks with a microwave communication link (MWL). The specifications of the MWL are to perform ground to space time and frequency comparisons with a stability of $0.3 \mathrm{ps}$ at one ISS pass and $7 \mathrm{ps}$ at one day. The ACES mission has applications in several domains such as fundamental physics, metrology or geodesy.

The raw measurements of the ACES MWL need to be related to the scientific products including all corrections (relativity, atmosphere, internal delays or phase ambiguities) and considering all terms greater than $0.1 \mathrm{ps}$ when maximized. In fact, the mission aims at extracting physical variables (scientific products) such as clock desynchronisation, electron content in the ionosphere (TEC), or range (instantaneous distance between the stations) from the code and phase measurements on ground and in space and auxiliary data (orbitography, internal delays, atmospheric parameters, ...).

To this purpose we have developed the complete model of the time transfer at the required 0.1 ps level. We have then developed in parallel two softwares:
\end{abstract}

Loïc Duchayne

LNE-SYRTE, Observatoire de Paris, LNE, CNRS, UPMC

61 av. de l'observatoire, 75014 Paris, France

E-mail: loic.duchayne@obspm.fr

Peter Wolf

LNE-SYRTE, Observatoire de Paris, LNE, CNRS, UPMC

61 av. de l'observatoire, 75014 Paris, France

Luigi Cacciapuoti

ESTEC, European Space Agency

Noordwijk, The Netherlands

Marc-Peter Hess

Astrium Space Transportation

Friedrichshafen, Germany

Marco Siccardi

TimeTech GmbH, Stuttgart, Germany

Wolfgang Schafer

TimeTech GmbH, Stuttgart, Germany 
1. a program to simulate the raw MWL measurements which allows the addition of different types of noise, biases, dead times in the measurements, phase ambiguities, etc $\cdots$

2. an algorithm which implements the MWL model and provides the "scientific products" from the raw measurements.

During the mission only the algorithm (2.) will be used, but the program (1.) is necessary for testing purposes. The two softwares are kept as independent as possible (different programming languages, different algorithms ...) to ensure a maximum efficiency of such tests. We provide some details on the software and the tests, considering different cases from the simplest to the more complex and realistic situation using real ISS orbitography data and MWL measurement noise from the MWL engineering model.

The phase ambiguity removal of carrier phase measurements is performed by the algorithm and its success strongly depends on the noise of the observables. We have investigated the statistics of cycle slips which appear during this operation using experimental data obtained from the tests of the MWL engineering model. We present two novel methods which allow the reduction of the cycle slip probabilities by a factor greater than 5 compared to the standard method.

\section{Introduction}

Due to recent scientific breakthroughs such as laser cooling and atom trapping methods, huge progress has been achieved on the uncertainties of atomic clocks during the last twenty years $[4,8, ?, ?]$. Some of them reach a precision of a few parts over $10^{17}$ in relative frequency.

In a Space environment these atomic sensors would become an exceptional tool for promising applications in fundamental physics, geodesy, time and frequency metrology or in navigation. Onboard terrestrial or solar system satellites, their exceptional properties allow them to test the fundamental laws of nature, to study the Earth's and solar system gravitational potential and its evolution, or to explore the Universe [21].

To this purpose, the ACES (Atomic Clock Ensemble in Space) mission [15], an ESACNES project will be installed onboard the ISS (International Space Station) in 2013. It consists of two atomic clocks and a two-way time transfer system (microwave link, MWL) with an overall uncertainty goal of 1 part in $10^{16}$ after ten day integration (see section 2 for more details). This mission will carry out tests of fundamental physics such as testing the Einstein's Equivalence Principle or perform time transfer at the sub-picosecond level.

This next generation of space clocks at the envisaged uncertainty level requires a fully relativistic analysis, not only of the clocks (in space and on the ground) but also of the time/frequency transfer method used to compare them [1, 10, 13, 20,5,11]. Similarly the modeling of the mission for the future data analysis requires a relativistic framework and the investigation of the complete model complying with the envisaged performances.

The preparation of the mission data processing needs the development of two softwares. On one hand, because the mission is still in preparation, we need to simulate the MWL raw measurements in the most realistic way possible. On the other hand, the mission requires an algorithm to extract the "scientific products" from the raw measurements and the parameters of the mission.

Finally, similarly to Global Positioning Systems, the phase ambiguity resolution is a key process which the mission success depends on. In fact an error of one cycle on one of the 
three frequencies will lead to an error larger than the mission specifications. The occurrence probability of cycle slips strongly depends on the raw measurement noise and observable combinations must be found to reduce this probability.

In this paper we study in more detail the preparation of the data analysis and provide some details on its testing which will allow to evaluate the high performances of the MWL. In sections 2 and 3 we briefly describe the ACES mission, and then the relativistic model used for the clocks and the time transfer to understand the nature of the raw measurements. We also relate their expression with all propagation effects between the two clocks (internal delays, atmospheric effects, relativistic contributions,...). This investigation is required to model the time transfer at the expected level. In section 4 we give information about the measurement simulation and the scientific products extracting algorithm and show some tests of the latter to evaluate its performances in terms of stability and accuracy. In the two last sections (5) and 6) before the conclusion, we discuss the phase ambiguity determination whose success strongly depends on the noise of the observables. We investigate the statistics of cycle slips which appear during this operation. To this purpose we use experimental data obtained from the tests of the MWL engineering model [16], and an ISS ephemeris.

\section{The ACES mission}

The ACES project led by the CNES and the ESA aims at setting up on the ISS several highly stable clocks around 2013. The ACES payload includes two clocks, a hydrogen maser (SHM developed by TEMEX) and a cold atom clock PHARAO (developed by CNES) respectively for short and long term performances, and a microwave link for communication and time/frequency comparison. The frequency stability of PHARAO onboard the ISS is expected to be better than $10^{-13}$ for one second, $3 \cdot 10^{-16}$ over one day and $1 \cdot 10^{-16}$ over ten days, with an accuracy goal of $1 \cdot 10^{-16}$ in relative frequency.

The ACES mission has as objectives :

- to operate a cold atom clock in microgravity with a $100 \mathrm{mHz}$ linewidth,

- to compare the high performances of the two atomic clocks in space (PHARAO and SHM) and to obtain a stability of $3 \cdot 10^{-16}$ over one day,

- to perform time comparisons between the two space clocks and ground clocks,

- to carry out tests of fundamental physics such as a gravitational redshift measurement and to search for a potential speed of light anisotropy and a possible drift of the fine structure constant $\alpha$.

- to perform precise measurements of the Total Electron Content (TEC) in the ionosphere, the tropospheric delay and the Newtonian potential.

The time transfer is performed using a micro-wave two-way system, called Micro-Wave Link (MWL). An additional frequency is added in order to measure and correct the ionospheric delay at the required level. It uses carriers of frequency $13.5,14.7$ et $2.25 \mathrm{GHz}$, modulated by pseudo random codes respectively at $10^{8} s^{-1}, 10^{8} s^{-1}$ and $10^{6} s^{-1}$ chip rates. Moreover it has four channels that allow four ground stations to be compared with the ISS clock at the same time.

According to the mission specifications, the microwave link has to synchronize two atomic clocks with a time stability of $\leq 0.3$ ps over $300 \mathrm{~s}, \leq 7 \mathrm{ps}$ over one day, and $\leq 23 \mathrm{ps}$ over 10 days. The performance of this link is a key issue since it will perform high precision time comparisons without damaging the high performances of the clocks. 
For our purposes we express the above requirements for the MWL in a simplified form by the temporal Allan deviation $\left(\sigma_{x}(\tau)\right)$ :

$$
\sigma_{x}(\tau)=5.2 \cdot 10^{-12} \cdot \tau^{-\frac{1}{2}}
$$

for a single satellite pass over a ground station (for integration times $\tau$ lower than $300 \mathrm{~s}$ ) and by

$$
\sigma_{x}(\tau)=2.4 \cdot 10^{-14} \cdot \tau^{\frac{1}{2}}
$$

for longer integration times (for integration times $\tau$ greater than $300 \mathrm{~s}$ ).

We take (1) and (2) as our upper limits for all comparisons with the test results of the data processing algorithm in the following sections.

\section{The time transfer model}

Due to the outstanding performances of the clocks, some effects from fundamental physics have an impact on the clock behaviors and on the signal propagation. In this situation, a relativistic point of view must be considered to model the clocks and the time transfer. The aim of this section is to describe the relativistic model used for the ACES mission and the Micro-Wave Link. It is necessary to express the raw measurements as functions of the clock desynchronisation and of all effects which affect the signal propagation (relativity, atmosphere, internal delays, phase ambiguities, etc...) at the expected level.

In a general relativistic framework each clock produces its own local proper time, in our case $\tau^{g}$ and $\tau^{s}$ for the ground and space clocks respectively. In order to model signal propagation between the ground and the space stations, we use a non-rotating geocentric spacetime coordinate system. Thus $t=x_{0} / c$ is the geocentric coordinate time, $\vec{x}=\left(x_{1}, x_{2}, x_{3}\right)$ are the spatial coordinates, where $\mathrm{c}$ is the speed of light in vacuum $\left(\mathrm{c}=299792458 \mathrm{~m} . \mathrm{s}^{-1}\right)$. We denote $U(t, \vec{x})$ as the total Newtonian potential at the coordinate time $t$ and the position $\vec{x}$ with the convention that $U \geq 0$ [19]. In these coordinates, the metric is given by an approximate solution of Einstein's equations valid for low velocity and potential $\left(\frac{U}{c^{2}}<<1\right.$ and $\left.\frac{v^{2}}{c^{2}}<<1\right)$ :

$$
d s^{2}=-\left(1-\frac{2 U(t, \vec{x})}{c^{2}}\right) c^{2} d t^{2}+\left(1+\frac{2 U(t, \vec{x})}{c^{2}}\right) d \vec{x}^{2}
$$

where higher order terms can be neglected for our purposes [20].

In this system, each emission or reception event (at the antenna phase center) is identified by its coordinate time $t_{i}$ (figure 5) and a coordinate time interval is defined by $T_{i j}=t_{j}-t_{i}$. We define $\vec{x}_{g}, \vec{v}_{g}$ and $\vec{a}_{g}$ respectively as the position, the velocity and the acceleration of the ground station, and $\vec{x}_{s}, \vec{v}_{s}$ and $\vec{a}_{s}$ respectively as the position, the velocity and the acceleration of the space station.

The $f_{1}$ frequency signal is emitted by the ground station at the coordinate time $t_{1}$ and received by the space station at $t_{2}$. The $f_{2}$ and $f_{3}$ frequency signals are emitted from the space station at $t_{3}$ and $t_{5}$, and received at the ground station at $t_{4}$ and $t_{6}$. The third frequency is added to measure the TEC in the ionosphere which allows the correction of the ionospheric delay.

According to equation (3), we introduce the notation $[.]^{A}$ to express an interval of the coordinate time $t$ in the proper time scale $\tau^{A}$ produced by a clock at $\vec{x}_{A}(t)$. The following 


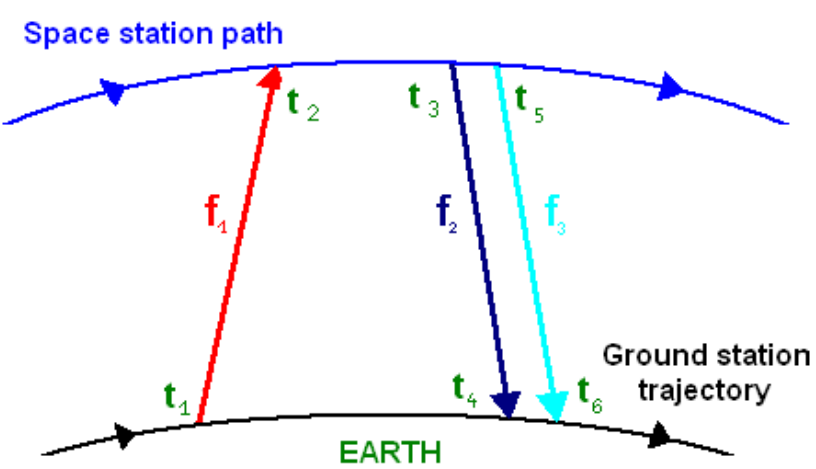

Fig. 1 MWL principle

equation relates a coordinate time interval $T_{12}$ elapsed between the coordinate time $t_{1}$ and $t_{2}$ to a proper time interval $\left[T_{12}\right]^{A}$ elapsed in the proper time scale $\tau^{A}$ :

$$
\left[T_{12}\right]^{A}=\int_{t_{1}}^{t_{2}}\left(1-\frac{U\left(t, \vec{x}_{A}\right)}{c^{2}}-\frac{v_{A}^{2}(t)}{2 c^{2}}\right) d t,
$$

where $\vec{x}_{A}$ and $v_{A}$ are respectively the position and the norm of the velocity of a point A expressed in the coordinate system described above, and the integral is evaluated along the path of the clock $\vec{x}_{A}(t)$.

The MWL is characterized by its continuous way of emission. It measures the time offsets between the locally generated signal and the received one. It provides three measurements (or observables) of the code (one on the space station, two on the ground) and three measurements of the phase of the carrier frequency at a sampling rate of one Hertz.

An observable is related to the phase comparison between a signal derived from the local oscillator and the received signal, corrected for the frequency difference mainly due to the first order Doppler effect (see [2] for details of a similar procedure used in GPS). If we consider a special bit of the signal which is produced locally at $\tau_{p}$ and received at $\tau_{a}$, an observable is a measurement of the local proper time interval between these two events. The observable is labeled with the arrival proper time $\tau_{a}$.

For instance, the upwards signal observable is

$$
\Delta \tau^{s}\left(\tau_{a}^{s}\right)=\tau_{p}^{s}-\tau_{a}^{s}=\tau_{p}^{g}-\tau_{a}^{s}
$$

The last identity comes from the fact that two identical segments of code are produced at the same local time : $\tau_{p}^{g}=\tau_{p}^{s}$.

Thus we have

$$
\Delta \tau^{s}\left(\tau^{s}\left(t_{2}\right)\right)=\tau^{g}\left(t_{1}\right)-\tau^{s}\left(t_{2}\right) .
$$

We define the desynchronisation between the two clocks at the coordinate time $t$ by the difference between the ground and the space proper times at $t, \tau^{g}(t)-\tau^{s}(t)$. The raw measurement can be expressed as a function of the desynchronisation between the two clocks at the time of reception $t_{2}$, the emission and reception internal delays, and the propagation time between the two stations $T_{12}$. For the sake of clarity and equation simplicity, we intentionally omit internal delays in the following expressions. They can be associated with the 
propagation time except the fact they are proper time intervals in the local clock time scale, and their inclusion in the model poses no particular difficulties.

For example, if we consider the observable from $f_{1}$ signal PRN code, it is given by

$$
\Delta \tau^{s}\left(\tau^{s}\left(t_{2}\right)\right)=\tau^{g}\left(t_{2}\right)-\tau^{s}\left(t_{2}\right)-\left[T_{12}\right]^{g} .
$$

where $T_{12}$ is the propagation time for the $f_{1}$ signal. The latter is a coordinate time interval transformed to a ground proper time interval following equation (4).

As we want to evaluate the desynchronisation between the two clocks, the time interval $T_{12}$ elapsed between emission from the ground station and reception by the satellite of the $f_{1}$ frequency signal needs to be calculated. It can be written as

$$
\begin{aligned}
T_{12} & =\frac{R_{12}}{c}+\frac{2 G M_{E}}{c^{3}} \ln \left(\frac{x_{g}\left(t_{1}\right)+x_{s}\left(t_{2}\right)+R_{12}}{x_{g}\left(t_{1}\right)+x_{s}\left(t_{2}\right)-R_{12}}\right) \\
& +\Delta_{12}^{\text {tropo }}+\Delta_{12}^{\text {iono }}+O\left(\frac{1}{c^{4}}\right),
\end{aligned}
$$

where $R_{12}=\left\|\overrightarrow{R_{12}}\right\|=\left\|\overrightarrow{x_{s}}\left(t_{2}\right)-\overrightarrow{x_{g}}\left(t_{1}\right)\right\|$, where the logarithmic term represents the Shapiro time delay [17] (see e.g. [5] for a detailed derivation) and where $\Delta_{12}^{\text {tropo }}$ and $\Delta_{12}^{\text {iono }}$ are respectively the tropospheric and ionospheric delays on the signal path.

The phase observable for the $f_{1}$ signal can be expressed similarly except for two important features : the effect of ionosphere and the phase ambiguity. The ionosphere delay takes opposite signs for code and phase [3]. Moreover a carrier phase measurement is less noisy than code measurement but affected by a phase ambiguity. This ambiguity corresponds to integer number $N_{i}$ of the signal period $1 / f_{i}$. For instance the phase measurement of the $f_{1}$ signal is given by

$$
\Delta \tau_{\phi}^{s}\left(\tau^{s}\left(t_{2}\right)\right)=\tau^{g}\left(t_{2}\right)-\tau^{s}\left(t_{2}\right)-\left[T_{12}^{\phi}\right]^{g}+N_{1} / f_{1}
$$

where $T_{12}^{\phi}$ is the phase propagation time of the $f_{1}$ signal and given by 8 with a change of sign of the ionospheric term $\Delta_{12}^{\text {iono }}$.

An error in the determination of $N_{i}$ is referred to as a "cycle slip". It will be studied in detail in sections (5) and (6).

The expressions of code and phase observables for the two remaining frequencies can be derived similarly to (7) and (9). The combination of $f_{1}$ and $f_{2}$ signal observables gives the expression of the desynchronisation between ground and space clocks :

$$
\begin{aligned}
\tau^{g}\left(t_{a}\right)-\tau^{s}\left(t_{a}\right) & =\frac{1}{2}\left(\Delta \tau^{s}\left(\tau^{s}\left(t_{2}\right)\right)-\Delta \tau^{g}\left(\tau^{g}\left(t_{4}\right)\right)\right. \\
& +T_{12}-T_{34} \\
& -\int_{t_{1}}^{t_{2}}\left(\frac{U\left(t, \overrightarrow{x_{g}}\right)}{c^{2}}+\frac{v_{g}^{2}(t)}{2 c^{2}}\right) d t \\
& \left.+\int_{t_{3}}^{t_{4}}\left(\frac{U\left(t, \overrightarrow{x_{s}}\right)}{c^{2}}+\frac{v_{s}^{2}(t)}{2 c^{2}}\right) d t\right),
\end{aligned}
$$


where $t_{a} \equiv \frac{t_{2}+t_{4}}{2}$, and where $\Delta \tau^{s}\left(\tau^{s}\left(t_{2}\right)\right)$ and $\Delta \tau^{g}\left(\tau^{g}\left(t_{4}\right)\right)$ are the observables respectively from the ground and onboard the satellite, and where we have neglected non-linearities of $\tau^{g}(t)$ and $\tau^{s}(t)$ over the interval $t_{4}-t_{2}$ (few milliseconds). The integral terms result from proper time to coordinate time transformations. They are small corrections of order $10^{-12} \mathrm{~s}$ to the desynchronisation $\tau^{g}\left(t_{a}\right)-\tau^{s}\left(t_{a}\right)$.

In (10), the difference $T_{12}-T_{34}$ needs to be calculated from the knowledge of satellite and ground positions and velocities obtained from orbit restitution (see [6]). It includes the determination of ionospheric delays through the evaluation of the Total Electron Content (TEC). This is done by combining the observables coming from $f_{2}$ and $f_{3}$ signals because the signal paths are approximatively the same and there is almost one order of magnitude between the two frequencies which provides a good estimation of the TEC.

The same equation exists for phase observables. Considering phase ambiguities are correctly removed, it differs from the previous equation (10) by the term $T_{12}-T_{34}$. This term is not the same for code and phase desynchronisation equation due to the different effects from ionosphere on signal propagation.

\section{Data analysis and simulation}

The data analysis of ACES measurements has specific goals. It aims at providing the searched scientific products from the MWL measurements and some additional inputs, in particular ISS orbitography, parameters for the troposphere model, etc... Its goal is to evaluate physical parameters which will serve to study the Earth's atmosphere or to probe the fundamental laws of physics.

However, the data analysis has to deal with experimental issues such as noise on measurements or loss of signal acquisition which leads to dead times in the observables. Even in adverse conditions, it has to provide results within the expected performances of the mission. Thus the data analysis must be prepared before the launch of the mission, and tested in all pessimistic situations.

To this purpose, two software packages have been developed considering the complete model of time transfer described in [6]. On one hand, a raw MWL measurement simulation which produces the observables from given clock difference, orbit, internal delays, ionosphere and troposphere parameters, and on the other hand, a data analysis algorithm which extracts the searched parameters from the raw measurements. The two softwares have been implemented in the aim of keeping them as independent as possible. That is why they use different languages of programmation and different algorithms of calculation.

In the following paragraphs we will give some details on both softwares.

\subsection{The raw measurement simulation}

The simulation produces the raw measurements of the MWL for code and carrier phase on the three frequencies. To this purpose it needs several parameters chosen by the user such as the clock behaviors $\left(\tau^{g}(t)\right.$ and $\left.\tau^{s}(t)\right)$, the space and ground station trajectories or the evolution of internal delays.

It is implemented in an object oriented language and simulates the six observables using an iteration procedure.

As an example, the simulation of the $f_{1}$ signal code measurement $\Delta \tau^{s}\left(\tau^{s}\left(t_{2}\right)\right)$ measured at the space proper time $\tau^{s}\left(t_{2}\right)$ starts with the calculation of the propagation time $T$ between 
the ground and space stations respectively located at $\overrightarrow{x_{g}}\left(t_{2}\right)$ and $\overrightarrow{x_{s}}\left(t_{2}\right)$, which are obtained by interpolation of the orbitography data. Due to the propagation delay, the ground station was not at $\overrightarrow{x_{g}}\left(t_{2}\right)$, but at a previous position along its trajectory when emitting the signal. New position, velocity and acceleration are interpolated at the coordinate time $t_{2}-T$ which allows to calculate a new value for the propagation time $T$. By convergence, this method provides the true time of signal emission $t_{1}$ by alternatively calculating the propagation time $T$ for a signal arriving at the coordinate time $t_{2}$ and interpolating the ground station position, velocity and acceleration. We then evaluate the proper time $\tau^{g}\left(t_{1}\right)$ of signal emission from the ground station and finally the code observable $\Delta \tau^{s}\left(\tau^{s}\left(t_{2}\right)\right)=\tau^{g}\left(t_{1}\right)-\tau^{s}\left(t_{2}\right)$.

Once the "theoretical" measurement is calculated, perturbations are added to it. The simulation allows addition of measurement noise, clock error, internal delays and errors on them, antenna phase patterns, dead times in measurements, orbitography error, etc ...

\subsection{The data processing algorithm}

The MWL data analysis implements the MWL model and provides the scientific products from the raw MWL measurements. Its outputs are the Total Electron Content (TEC) in the ionosphere along the line of sight, the desynchronisation between the ground and the space clocks, the tropospheric delay and the instantaneous distance between the two stations. These products are obtained from code only, and code plus phase measurements. Besides the raw measurements, the calculation needs some other data such as the orbitography of the ground and space stations, atmospheric parameters (temperature, pressure, ...) or the internal delays.

Contrary to the raw measurement simulation, the data processing algorithm is written in an assembly language. Before the calculation of the scientific products, it performs a preprocessing so as to detect dead times in code and phase measurements, to combine correctly the measurements of the three frequencies and to transform space and ground station trajectories in the adapted coordinate system. Moreover it also determines cycle ambiguities on the carrier phase measurements before using the non-ambiguous phase observables to estimate the scientific products.

The calculation is based on analytical expressions at a chosen time. For instance, in expression (10), the term $T_{12}-T_{34}$ is evaluated with a Taylor expansion. Its expression is a function of position, velocity and acceleration of the two stations at one chosen coordinate time. The same kind of expansions are used for the calculation of TEC, range or tropospheric delay.

\subsection{Tests and results}

The testing of the algorithm is performed by taking into account independently and then simultaneously the different noises and perturbations which will appear during the mission (code and phase measurements noise, clock noise, orbit restitution noise, dead times, ...). It allows to verify its stability and accuracy performances.

To this purpose we feed the output of the raw measurement simulation into the data analysis algorithm and compare if its output corresponds to the initial given functions used as input for the simulation. The differences between the outputs of the algorithm and the initial functions are identified as final errors on scientific products. In the actual mission, only the MWL data analysis will be used, to obtain directly the required scientific products. 


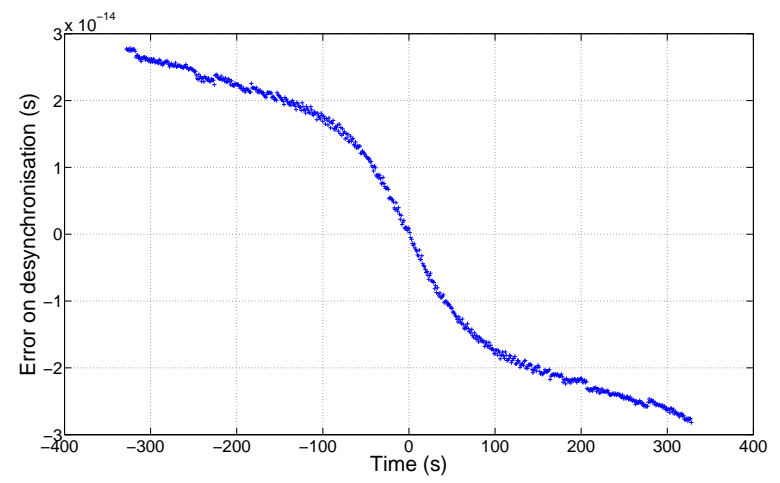

Fig. 2 Error on desynchronisation when no noise is added

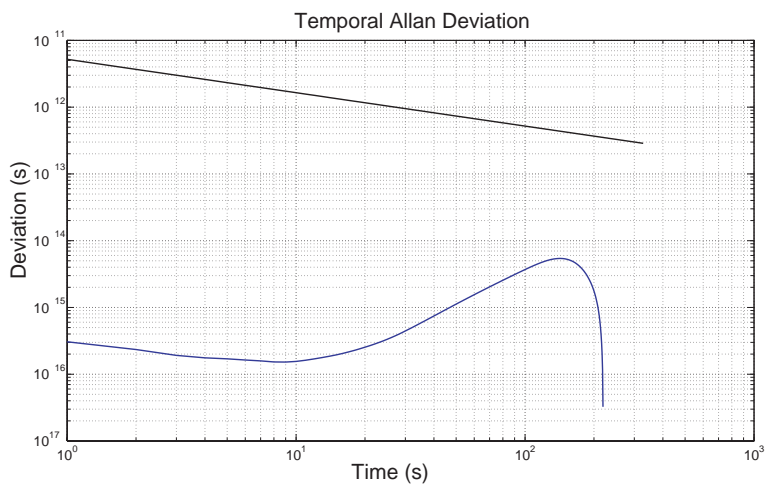

Fig. 3 Temporal Allan deviation when no noise is added

Many tests have been carried out, but for clarity, we only show the results of three of them. First we add no noise which would damage the performances of the link. The obtained error on desynchronisation is drawn on figure 2 and stays under 0.1 picoseconds. The remaining term corresponds to difference of tropospheric and ionospheric delays for $\mathrm{Ku}$ signals $\left(f_{1}\right.$ and $\left.f_{2}\right)$ which have not crossed the same atmospheric layers. The resulting deviation of the error on the desynchronisation is two orders of magnitude below the specifications (cf. figure 3). We conclude that no term giving a deviation over the specifications has been neglected.

Now the three observables have dead time intervals. They can be caused for example by a loss of station visibility or to the occultation of the reception antenna by a solar panel. Figure 4 shows that the software deals with dead times and provides the scientific products without error. The corresponding deviation is also two orders of magnitude under the specifications of the mission.

Finally the last presented test is performed adding white noise on all simulated raw measurements with an amplitude corresponding to the specifications (1). The resulting deviation stays under the specifications (cf. figure 5). It is calculated in accordance with equation (10) : the white noises from $f_{1}$ and $f_{2}$ observables are not correlated which explains the square root of two gain with respect to the mission specifications. The addition of noise is han- 


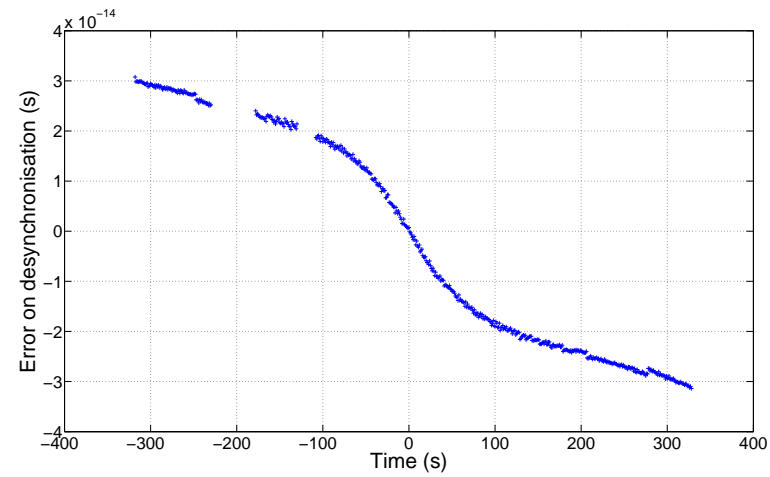

Fig. 4 Error on desynchronisation with dead times in measurements

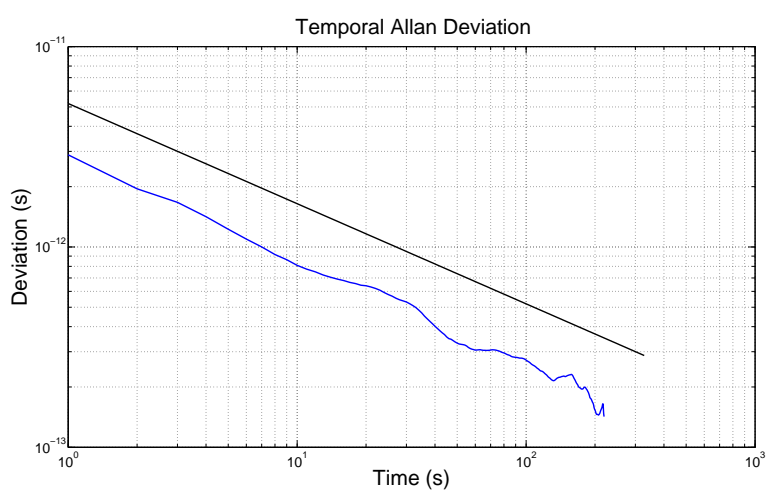

Fig. 5 Temporal Allan deviation considering white measurement noise on observables

dled by the data analysis software and this is demonstrating the robustness of the algorithm confronting this difficulty.

As written before, many other successful tests have been done considering different perturbations affecting the accuracy and the stability of the ACES time transfer. They involve realistic orbitography error, random walk noise on clocks or offset and noise on internal delays. The algorithm survived all these tests which complies with its initial objectives. In addition, the combination of the two softwares allows one to easily evaluate the effects of each parameter on the time and frequency transfer or to find out what the final results would be if there was an Einstein Equivalence Principe violation.

\section{Phase ambiguity resolution}

For all time/frequency transfer techniques the carrier phase observables have the advantage of significantly better precision than the code measurements, and therefore their use has been implemented in most techniques. Using the phase observables requires resolution of the phase ambiguities, which has been investigated in detail in many techniques, in particular the Global Positioning System [7,9]. 
However there are several differences between GPS and the MicroWave Link. On one hand, the MWL is a two way time transfer with three frequencies involved. The combination of measurements coming from an upwards and a downwards signal allows to eliminate restraining factors without canceling the clock behaviors. On the other hand, the frequencies used in the MWL are higher than GPS frequencies except for the third MWL frequency which is added to evaluate the TEC in the ionosphere. There is almost one order of magnitude between the lowest and the highest frequencies. The phase ambiguity identification must therefore be investigated for the particular case of the ACES mission.

Similarly to equation (10), the desynchronsation between the two clocks can be expressed as a function of phase observables and cycle ambiguities. It allows to study the consequences of the phase ambiguity detection on the final calculation.

$$
\begin{aligned}
\tau^{g}\left(t_{a}\right)-\tau^{s}\left(t_{a}\right) & =\frac{1}{2}\left(\Delta \tau_{\phi}^{s}\left(\tau^{s}\left(t_{2}\right)\right)-\Delta \tau_{\phi}^{g}\left(\tau^{g}\left(t_{4}\right)\right)-\frac{N_{1}}{f_{1}}\right. \\
& +\frac{N_{2}}{f_{2}}+\Delta-\left(\frac{1}{f_{1}^{2}}-\frac{1}{f_{2}^{2}}\right) \frac{f_{2}^{2} f_{3}^{2}}{f_{2}^{2}-f_{3}^{2}} \times \\
& \left.\left(\Delta \tau_{\phi}^{s}\left(\tau^{s}\left(t_{4}\right)\right)-\Delta \tau_{\phi}^{g}\left(\tau^{g}\left(t_{6}\right)\right)-\frac{N_{2}}{f_{2}}+\frac{N_{3}}{f_{3}}\right)\right),
\end{aligned}
$$

where the last term is the correction for the leading order ionospheric effect and $\Delta$ includes terms due to path asymmetries, tropospheric delays, relativistic corrections, higher order ionospheric terms, etc... (see [6] for details). Although these terms need to be taken into account, they are not relevant for the discussion concerning phase ambiguity resolution and will be neglected in the following.

According to equation (11), the cycle slip detection is necessary for the three frequencies. In fact an error of one cycle on $f_{1}, f_{2}$ and $f_{3}$ (i.e. an error of one unit in the determination of $N_{1}, N_{2}$ and $N_{3}$ ) leads respectively to an error of $3.7 \cdot 10^{-11} \mathrm{~s}, 3.4 \cdot 10^{-11} \mathrm{~s}$ and $1.0 \cdot 10^{-12} \mathrm{~s}$ on the clock desynchronisation, significantly larger than the uncertainties aimed at.

The carrier phase ambiguity $N_{i}$ on the $f_{i}$ phase observable is evaluated by combining the phase and code measurements and fixing $N_{i}$ to the nearest integer. These unknown numbers of periods added to phase measurements stay constant for continuous data ie. as long as the signal acquisition is kept. This means we obtain during a pass several evaluations of the same $N_{i}$ and then we can average the noise on them to get a more accurate value. Consequently correct cycle slip identification depends strongly on the observable noise types and levels.

In order to express the phase ambiguities as a combination of observables, we have to use a number of hypothesis. First the path of code and phase measurements tagged with the same coordinate time and at the same frequency are identical. We neglect the change on geometric delays due to the opposite effect of ionosphere on code and phase propagations. Moreover we assume all downwards signals received at the same coordinate time by the ground station have the same path through the atmosphere. Finally the part of ionosphere crossed by upwards and downwards signals are supposed identical for combined observables (i.e. for signals within a few ms of each other).

When forming the difference between phase and code observables measured at the same coordinate time and at the same frequency, we cancel all the propagation terms except the ionospheric delay (see equations (7), (8) and (9)). Actually the ionospheric term depends 
on the signal frequency and on the measurement nature, code or carrier phase. The leading term is proportional to the inverse of the square of the considered frequency. In addition, for the same frequency, the effect of ionosphere on code is at first order the opposite effect of ionosphere on carrier phase. The error on the ionospheric delay comes from the error on the TEC determination and is dominated by its leading term. Then the combination of equations (7) and (9) for the ambiguity $N_{1}$ on the first frequency can be written as :

$$
\frac{N_{1}}{f_{1}}=\Delta \tau_{\phi}^{s}\left(\tau^{s}\left(t_{2}\right)\right)-\Delta \tau^{s}\left(\tau^{s}\left(t_{2}\right)\right)-2 \cdot \frac{40.308}{c f_{1}^{2}} T E C .
$$

The same kind of equation can be written for $f_{2}$ using code and phase observables, $\Delta \tau^{g}\left(\tau^{g}\left(t_{4}\right)\right)$ and $\Delta \tau_{\phi}^{g}\left(\tau^{g}\left(t_{4}\right)\right)$, or for the third frequency $f_{3}$ with $\Delta \tau^{g}\left(\tau^{g}\left(t_{6}\right)\right)$ and $\Delta \tau_{\phi}^{g}\left(\tau^{g}\left(t_{6}\right)\right)$. They relate the phase ambiguity $N_{i}$ with the difference between phase and code observables and twice the ionospheric delay at the considered frequency. Consequently the error on the phase ambiguity $\delta N_{i}$ depends on the code and phase measurement noises, respectively $\delta C_{i}$ and $\delta \Phi_{i}$, and on the error on the TEC evaluation. The latter is determined with the code measurements from $f_{2}$ and $f_{3}$ signals [6]:

$$
\frac{40.308}{c} T E C=\frac{f_{2}^{2} f_{3}^{2}}{f_{2}^{2}-f_{3}^{2}}\left(\Delta \tau^{g}\left(\tau^{g}\left(t_{4}\right)\right)-\Delta \tau^{g}\left(\tau^{g}\left(t_{6}\right)\right)\right),
$$

The Total Electron Content is evaluated with this formula and the error on its determination is dominated by $f_{2}$ and $f_{3}$ code measurement error. Hence we obtain, for each frequency $f_{i}$, the dependence of the error on the phase ambiguity $\delta N_{i}$ on the uncertainty in the observables by combining equations (12) and (13):

$$
\begin{gathered}
\text { for } f_{1}: \frac{\delta N_{1}}{f_{1}}=\delta \Phi_{1}-\delta C_{1}-5.7 \cdot 10^{-2}\left(\delta C_{2}-\delta C_{3}\right), \\
\text { for } f_{2}: \frac{\delta N_{2}}{f_{2}}=\delta \Phi_{2}-\delta C_{2}-4.8 \cdot 10^{-2}\left(\delta C_{2}-\delta C_{3}\right), \\
\text { for } f_{3}: \frac{\delta N_{3}}{f_{3}}=\delta \Phi_{3}-\delta C_{3}-2.04 \cdot\left(\delta C_{2}-\delta C_{3}\right) .
\end{gathered}
$$

In the last equation 16 , two terms involve the $f_{3}$ code noise $\delta C_{3}$. They are perfectly correlated and partially cancel each other.

The noise model is evaluated with the latest results from the engineering model [16]. These results correspond to code and phase measurements performed on a $f_{1}$ signal for a received power of $-95 \mathrm{dBm}$ (this $\mathrm{S} / \mathrm{N}$ ratio is related to a zenithal position of the ISS over the ground station at about $350 \mathrm{~km}$ ). They show respectively a standard deviation of $1 \cdot 10^{-12} \mathrm{~s}$ and $1 \cdot 10^{-13} \mathrm{~s}$ for the code and the phase measurements. This $1 \mathrm{~s}$ noise will be used in this section to estimate the statistics of cycle slips.

For this purpose, we assume the noise on $f_{2}$ approximatively the same. On the contrary, the noise on $f_{3}$ is assumed to be $7\left(\simeq f_{1} / f_{3}\right)$ times larger on the carrier phase and 100 (ratio of chip rates) times larger on the code. In the following table (Tab.11), we give the $2 \sigma$ uncertainty (at $1 \mathrm{~s}$ points with no averaging) of each term appearing in the equations (14), (15) and (16). These noise levels have to be compared with the half of the period $1 / f_{i}$ due to the fixing of the ambiguity $N_{i}$ to the nearest integer : if the $2 \sigma$ noise level is equal to half the period, there is a $5 \%$ chance of a cycle slip.

We note that at all frequencies the cycle slip probability is less than $5 \%$-remember that on $f_{3}$ the code noise and half the ionosphere noise cancel (see equation (16) ) - even when 
Table $195 \%$ confidence ( $2 \sigma$ uncertainty) of terms appearing in the phase ambiguity resolution according to equations (14), 15] and (16) for an input power of $-95 \mathrm{dBm}$

\begin{tabular}{|c|c|c|c|}
\hline $95 \%$ confidence $(2 \sigma$ uncertainty) & $f_{1}$ & $f_{2}$ & $f_{3}$ \\
\hline Noise on code $(s)$ & $2 \cdot 10^{-12}$ & $2 \cdot 10^{-12}$ & $2 \cdot 10^{-10}$ \\
Noise on carrier phase $(s)$ & $2 \cdot 10^{-13}$ & $2 \cdot 10^{-13}$ & $1.4 \cdot 10^{-12}$ \\
Ionospheric error due to the noise on $f_{3}$ code $(\mathrm{s})$ & $1.1 \cdot 10^{-11}$ & $9.6 \cdot 10^{-12}$ & $2 \cdot 2 \cdot 10^{-10}$ \\
Signal period $1 / f_{i}(\mathrm{~s})$ & $7.4 \cdot 10^{-11}$ & $6.7 \cdot 10^{-11}$ & $4.5 \cdot 10^{-10}$ \\
\hline
\end{tabular}

using individual $1 \mathrm{~s}$ measurements (no averaging). The probability can be further reduced by averaging the measurements of a continuous observation.

However two facts will disturb the cycle slip identification. First the received power does not remain at $-95 \mathrm{dBm}$ but decreases when the distance between the two stations increases. As an example, when the space station is at a ten degrees elevation, the received signal power is $-115 \mathrm{dBm}$. From the tests of the engineering model, this corresponds to an eight fold increase of the code noise. In these worst conditions, the direct method of ambiguity resolution fails. One solution is to carry out a weighted average with all $1 \mathrm{~s}$ measurements of a given continuous pass. Indeed, the noise level of each measurement will depend on the incoming power (varying as a function of elevation). The dependence of the noise on power has been measured so the weighting can be preformed as a function of the locally measured power level.

Secondly the measurement noise is not perfectly white. Some flicker noise damages the averaging and the ambiguity removal, and even averaging over the complete pass is insufficient due to the presence of flicker noise. We need to search for observable combinations to increase the ambiguity resolution success rate.

The figures of Tab. 1 show that the leading error terms come from the code noise on the third frequency. Our aim is to get rid of the code measurement of $f_{3}$, or at least to reduce its impact on the final statistics.

We start by noting that, according to the noise levels in Tab.1 the ambiguity resolution for the $\mathrm{Ku}$ signals $\left(f_{1}\right.$ and $\left.f_{2}\right)$ is more likely to be successful than for the third frequency. Then, if we suppose these ambiguity resolutions are correctly performed (this is likely the case, see section 6), these considerations bring two new methods for the $f_{3}$ signal ambiguity resolution. On the one hand, the combination of $f_{2}$ and $f_{3}$ phase measurements gives a new expression of $N_{3}$ which does not depend on the $f_{3}$ code measurement :

$$
\begin{aligned}
\frac{N_{3}}{f_{3}} & =\Delta \tau_{\phi}^{g}\left(\tau^{g}\left(t_{6}\right)\right)-\Delta \tau_{\phi}^{g}\left(\tau^{g}\left(t_{4}\right)\right) \\
& +\frac{N_{2}}{f_{2}}+\frac{f_{2}^{2}-f_{3}^{2}}{f_{2}^{2} f_{3}^{2}} \frac{40.308}{c} T E C .
\end{aligned}
$$

In the previous equation, the Total Electron Content is evaluated by the combination of $f_{2}$ phase and code measurements :

$$
\frac{40.308}{c} T E C=\frac{f_{2}^{2}}{2}\left(\Delta \tau_{\phi}^{g}\left(\tau^{g}\left(t_{4}\right)\right)-\Delta \tau^{g}\left(\tau^{g}\left(t_{4}\right)\right)-\frac{N_{2}}{f_{2}}\right) .
$$

It allows a new evaluation of the TEC whose error depends only on $\delta C_{2}$ and $\delta \Phi_{2}$ and which can replace the equation (13). Inserting equation (18) into (17) leads to 


$$
\frac{\delta N_{3}}{f_{3}}=\delta \Phi_{3}-22.72 \cdot \delta \Phi_{2}+21.72 \cdot \delta C_{2} .
$$

On the other hand, the second method is to substitute (13) into (12) (but written for $f_{3}$ ) for half the ionospheric term and using $(18)$ for the other half. The error from the $f_{3}$ code measurement almost cancels, and the error on $N_{3}$ is :

$$
\frac{\delta N_{3}}{f_{3}}=\delta \Phi_{3}+2.3 \cdot 10^{-2} \cdot \delta C_{3}-22.22 \cdot \delta \Phi_{2}+21.20 \cdot \delta C_{2} .
$$

Equations (19) and (20) reduce the error from the code measurements from $f_{3}$ and then increase the success rate. The choice between one of these methods depends on the real mission noise levels. With the noise measured in the engineering model tests, they both approximatively give a five fold gain on the uncertainty of $\delta N_{3}$.

In conclusion, the statistics of successful carrier phase ambiguity resolution strongly depend on the pass characteristics and its relation with the noise levels. These kind of realistic received noises are studied in the following section 6

\section{Tests with expected noise}

In this section, we investigate the error on the phase ambiguity resolution from realistic measurement noises and try to reduce the probability of cycle slips on the ambiguity identification. For an ISS pass over a ground station -ie. for time duration less than $600 \mathrm{~s}$-, the noise measured on the Engineering model can be modelled in a simplified from by its temporal Allan deviation. We use respectively for code and phase measurements at $-95 \mathrm{dBm}$ $\left(\sigma_{x}^{c}(\tau)\right)^{2}=\left(1 \cdot 10^{-12} \tau^{-\frac{1}{2}}\right)^{2}+\left(2 \cdot 10^{-13}\right)^{2} s$ and $\left(\sigma_{x}^{\phi}(\tau)\right)^{2}=\left(1 \cdot 10^{-13} \tau^{-\frac{1}{2}}\right)^{2}+\left(7 \cdot 10^{-14}\right)^{2} s$, where $\tau$ is the integration time [16].

The measured noise is composed of white noise and flicker noise. The former is averaged rapidly as the square root of time. Then the noise average is limited by the flicker noise. On figure (6), we compare the averaging of the measured noise on $f_{1}$ code at $-95 \mathrm{dBm}$, with the averaging of a pure white noise with the same level at one second $\left(\sigma=1 \cdot 10^{-12} \mathrm{~s}\right)$.

During a pass of the International Space Station over a ground station, the signal over noise ratio strongly varies and its received signal power can decrease by over $20 \mathrm{dBm}$. This ratio actually depends on the distance between the two stations, the elevation of ISS and others parameters (antenna orientation, weather conditions, etc ...). A model provided by EADS-Astrium gives power evolution with distance and elevation. The engineering model measurements of the noise dependence on power carried out at TimeTech [16] then allow us to derive the dependence of the noise on elevation and distance, i.e. the variation of the noise during a given pass.

We perform Monte Carlo simulations to estimate the probabilities of a cycle slip and its uncertainties ( $95 \%$ confidence). In fact, we create code and phase signals with the same noise properties as the measured noise on the engineering model for the frequency $f_{1}$. The $f_{2}$ and $f_{3}$ noise for code and phase measurements are similarly generated taking into account chip rate or frequency ratios. Then these noises are modulated as a function of the elevation and distance for a given pass. In a pessimistic approach we suppose the two kinds of noise (white and flicker) on code and phase measurements are affected in the same way by the received power. Hence we can calculate the $95 \%$ confidence of the ambiguity error in accordance with equations (14), (15), (16), (19) and (20). 


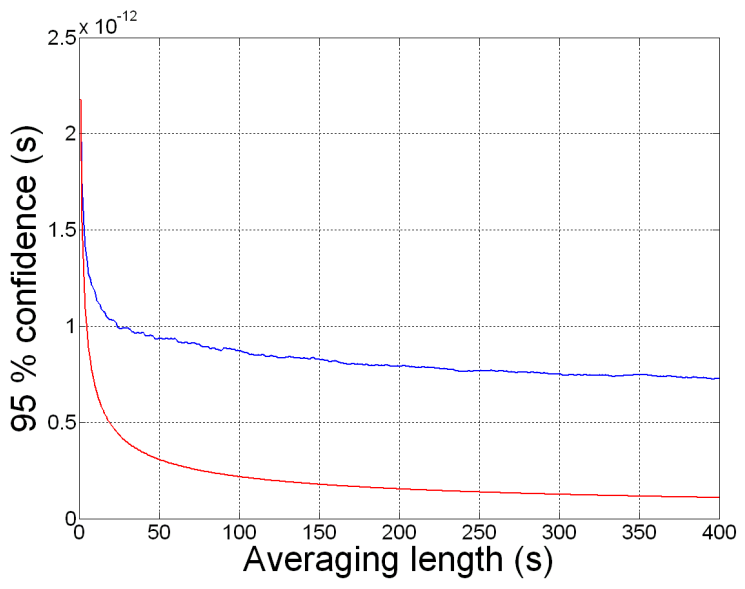

Fig. $695 \%$ confidence of averaging of white noise (red) and $f_{1}$ code measured noise at $-95 \mathrm{dBm}$ (blue). Both have the same deviation at one second.

The noise on the ambiguity identification is expressed in seconds : as this noise has to be compared with the signal period, we express it in term of a fraction $\delta N_{i}$ of the considered signal cycle. We average over a number of $1 \mathrm{~s}$ points to decrease the uncertainty on $N_{i}$. Then, if $\delta N_{i}$ after averaging exceeds half a cycle with more than $5 \%$ probability, we consider that the ambiguity resolution has failed as $N_{i}$ is fixed to the wrong number. As an improvement over simple averaging, we also consider weighted averaging, where the weights are determined by the noise level of each point (depending on received power, ie. elevation and distance).

In the following we study the time transfer between the International Space Station and a ground station based in Toulouse, France $\left(43^{\circ} 36^{\prime} N, 1^{\circ} 26^{\prime} E\right)$ to simulate realistic noise levels. Actually, this station has been chosen as the master ground station of the ACES mission. Then we choose an ISS trajectory corresponding to one pass over the ground station. To this purpose, we consider an ephemeris of ISS corresponding to the $20^{\text {th }}$ of May, 2005. However in order to simulate several passes with different elevation higher than four degrees, we slightly shift the time origin of the ground station trajectory. This way we obtain numerous passes characterized by their reached maximum elevation and whose duration is between 350 and $600 \mathrm{~s}$.

Figure (7) shows the uncertainties (95\% confidence) of $\delta N_{1}$ and $\delta N_{2}$ as a function of the maximal elevation reached by the space station during the pass. These uncertainties are calculated with and without a weighted averaging over a complete pass ie. data are acquired continuously during the pass. It allows to assess the effect of weighting on $N_{1}$ and $N_{2}$ final estimation.

Weighted averaging leads to a gain on the success rate and particularly with high elevations : at ninety degrees elevation, the statistics are almost divided by a factor six between the two averaging methods. In fact, at lower elevations, the noise level on the observables does not sensibly change during the pass, and in this case the weighting becomes inefficient. We note that above six degrees maximum elevation, the phase ambiguity is correctly determined (95\% confidence).

Finally we investigate the impact of averaging (arithmetic or weighted) and calculation (in accordance with equations (16), (19) or (20)) methods on the uncertainty on the $f_{3}$ phase 


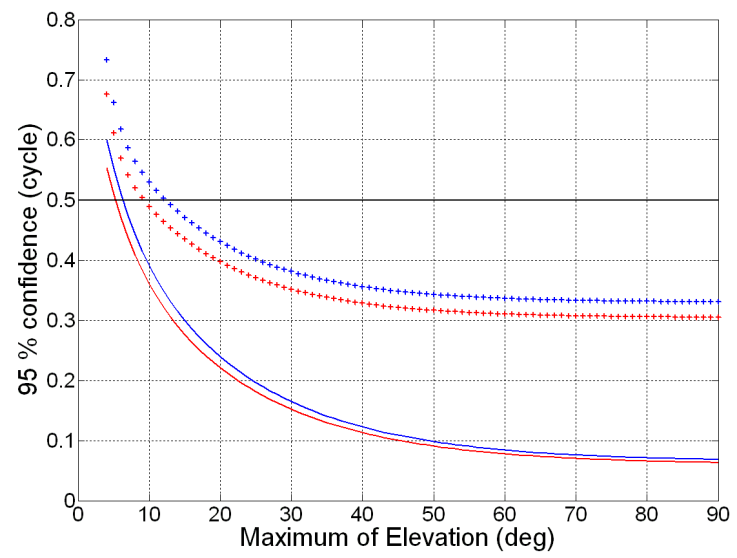

Fig. $795 \%$ confidence of the phase ambiguity resolution error as a function of the maximal elevation of the pass. The four curves show this dependence for $f_{1}$ (blue) and $f_{2}$ (red) signals using arithmetic (cross-dashed) or weighted (solid) averaging in accordance with equations 14 , and [15].

ambiguity $N_{3}$. For the noise considered in this paper, the methods coming from equations (19) and 20) give approximatively identical results.

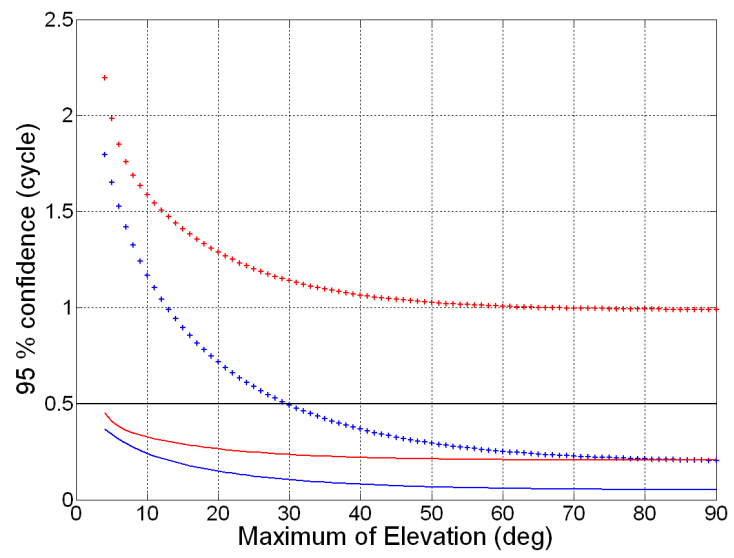

Fig. $895 \%$ confidence of the phase ambiguity resolution error for the $f_{3}$ signal as a function of the maximal elevation of the pass. The four curves show this evolution whether we consider first equation (16) (crossdashed) or proposed method (19) or 201) (solid) and whether arithmetic (red) or weighted (blue) averaging is performed.

Figure 8 depicts the different combination of approaches concerning the averaging and the calculation. Contrary to weighting, the observable combinations given by equation (19) or (20) brings a factor five gain for all elevations (see the two lowest curves on figure 8 ). We also remark that the proposed measurement combinations (19) or (20) are essential in the cycle slip detection on the third frequency, and thus for the mission objectives. 
However the obtained probability of successful ambiguity resolution on $f_{3}$ needs correct identification of the $\mathrm{Ku}$ cycle slips $\left(f_{1}\right.$ and $\left.f_{2}\right)$. Consequently, an unbiased estimation of the scientific products is not limited anymore by the $f_{3}$ phase ambiguity measurement but by the two first frequencies. But in any case, using weighted averaging and methods 19 and (20) for $f_{3}$, the cycle ambiguity resolution has less than $1 \%$ probability to fail for any uninterrupted satellite pass with a maximum elevation over four degrees if we suppose that $f_{1}$ and $f_{2}$ phase ambiguities have been correctly solved.

\section{Discussion and conclusion}

The models of the time transfer of the ACES mission and the associated relativistic effects were investigated at the 0.1 picosecond level. In addition, the measurements were related to all physical effects appearing during the station communication.

Then these models were applied to develop an end-to-end simulation. In fact, two softwares were implemented : a simulation which creates the raw measurements of the mission and allows addition of different kinds of perturbations, and an algorithm which uses these raw measurements to extract scientific products.

Furthermore the MWL phase ambiguity resolution was tackled and methods were studied to reduce the failure rate of this process. The cycle slip removal faced two difficulties, the presence of Flicker noise which can not be averaged, and the evolution of the signal over noise ratio during the pass. Considering a model for the power evolution with distance and elevation, we simulated noises complying with experimental code and phase measurements on the first frequency $f_{1}$. Then the $f_{2}$ and $f_{3}$ code and phase noises were deduced assuming they are related to $f_{1}$ code and phase noise with respectively chip rate and frequency ratios. In a pessimistic approach, we supposed all components of noise (white and Flicker) are similarly modulated by the $\mathrm{S} / \mathrm{N}$ ratio.

With Monte Carlo simulations, we obtained the most realistic estimates of the resulting performance for uninterrupted passes. The success rate is now limited by the ambiguity resolution of the Ku-band signals. In fact the high probability of successful resolution on the third frequency is based on the hypothesis that there is no cycle slip on $f_{1}$ and $f_{2}$. Their resolutions are limited by the code noise from frequency $f_{3}$. The failed resolution proportion for Ku-band signals is less than $5 \%$ for passes with maximum elevation over six degrees. Consequently, in this case, the phase ambiguity resolution is successful for all three frequencies with more than $94 \%$ probability. An increase of the S-band signal chip rate, from $10^{6} s^{-1}$ to $5 \cdot 10^{6} s^{-1}$, would strongly improve the Ku-band signal phase ambiguity as it will approximatively divide $f_{3}$ code noise by a factor 5 .

On one hand, these statistics are based on a pessimistic hypothesis of noise variation with received power. We assume indeed all kind of noise in the measurement are similarly modulated by the power evolution whereas it is likely that the Flicker noise is not or less affected. On the other hand, we have optimistically considered only completed uninterrupted passes over the ground station. In a close future, we will investigate the phase ambiguity resolution for incomplete passes (loss of lock during the pass) and other perturbations affecting the raw measurements during a pass (eg. temperature variations).

\section{Acknowledgements}

The research project is supported by the French space agency CNES, ESA and EADSAstrium through Loïc Duchayne's research scholarship $N^{o}$ 05/0812. 


\section{References}

1. D.W. Allan and N. Ashby, in: Kovalevsky J., Brumberg V.A. (eds.), Relativity in Celestial Mechanics and Astronomy. Proceedings of the IAU Symposium No. 114 Leningrad 1985, Reidel Publ., Dordrecht (1986).

2. T. B. Bahder, Phys. Rev. D, 68, 063005 (2003).

3. S. Bassiri and G. A. Hajj, ISSN 0065-3438, p. 1071-1086 (1993).

4. S. Bize et al., J. Phys. B: At. Mol. Opt. Phys., 38, S449 (2005).

5. L. Blanchet et al., Astronomy and Astrophysics, 370, 320 (2001).

6. L. Duchayne, F. Mercier, and P. Wolf, submitted to Astronomy \& Astrophysics, available on Arxiv : http://arxiv.org/abs/0708.2387

7. H-J. Euler and H. Landau, Proceedings of the 6th Int. Geodetic Symposium on Satellite Positionning, p. 650-659, 1992

8. T. P. Heavner et al., Metrologia, 42, 411 (2005).

9. P.J. de Jonge and C.C.J.M. Tiberius, vol. 12 of LGR-Series, Delft Geodetic Computing Centre, 1996

10. S. A. Klioner, Celestial Mechanics and Dynamical Astronomy, 53, 81 (1992).

11. B. Linet and P. Teyssandier, Phys. Rev. D, 66, 024045 (2002).

12. W. H. Oskay et al., Phys. Rev. Lett., 97, 020845 (2006).

13. G. Petit and P. Wolf, Astronomy and Astrophysics, 286, 971 (1994).

14. T. Rosenband et al., Science, 319, 1808 (2008).

15. C. Salomon et al., Cold atoms in space and atomic clocks : ACES, Comptes rendus de l'Acadmie des sciences. Srie IV, Physique, astrophysique, vol. 2, no9, pp. 1313-1330 (2001).

16. W. Schaefer et al., The ACES Microwave Link: instrument design and test results, proceedings of EFTF 2008.

17. I. I. Shapiro, Phys. Rev. Lett., 13, 789-791 (1964).

18. M. Soffel et al., Manuscripta Geodetica, 13143 (1988).

19. M. Soffel et al., Astronomical Journal, 126, 2687 (2003).

20. P. Wolf and G. Petit, Astronomy and Astrophysics, 304, 653 (1995).

21. P. Wolf et al., Quantum Physics Exploring Gravity in the Outer Solar System: The Sagas Project, submitted to Experimental Astronomy, available on Arxiv : http://arxiv.org/abs/0711.0304 (2007). 